\title{
Hausdorff Dimension Estimates for Invariant Sets of Time-Dependent Vector Fields
}

\author{
A. Noack and V. Reitmann
}

\begin{abstract}
In this paper we are concerned with generalizations of the results of A. Douady and J. Oesterlé [4] on estimates for the Hausdorff dimension of sets on Riemannian manifolds being negatively invariant with respect to a map. The main theorem that we derive for maps allows a number of corollaries which generalize several other results of $\mathrm{A}$. V. Boichenko, $\mathrm{F}$. Ledrappier and G. A. Leonov (see $[2,7,8]$ ). We extend the concept on differential equations and the corresponding vector fields on Riemannian manifolds. To obtain upper bounds for the Hausdorff dimension we formulate conditions for the eigenvalues of the symmetric part of the covariant derivative of the vector field. Modifications of the eigenvalues by the choice of an apropriate Riemannian metric will be of great importance. Besides the investigation of dimension of negatively invariant sets we are interested in the convergence behaviour of autonomous differential equations on Riemannian manifolds. We propose also a general, form of the Bendixson-Dulac criterion for the non-existence of non-trivial periodic orbits of vector fields on compact Riemannian manifolds.
\end{abstract}

Keywords: Riemannian manifolds, Hausdorff dimension estimates, dynamical systems, global convergence, Bendixson-Dulac criterion

AMS subject classiflcation: Primary 58 F 12, 58 F 39, secondary 58 B 20, 58 C 25

\section{Introduction}

In the papers $[2,8]$ generalizations of a theorem by A. Douady and J. Oesterlé [4] about estimations of the Hausdorff dimension of negatively sets invariant with respect to a map are obtained by requiring weaker conditions for the map under consideration. Lyapunov functions are introduced to modify the Jacobian matrix of the tangent map. That includes naturally a change of the singular values of the Jacobian matrix.

In Section 2 of this paper we show that the results published in $[2 ; 8]$ follow directly from a generalization of the theorem of A. Douady and J. Oesterle for Riemannian manifolds. With slightly stronger assumptions such kind of generalization is quoted in [7].

Supplementary to the results of $[2,4,7,8]$ in the present paper we give estimates of the Hausdorff dimension for sets which are negatively invariant with respect to shift

A. Noack: Techn. Univ. Dresden, Inst. Analysis, Mommsenstr. 13, D - 01062 Dresden V. Reitmann: Techn. Univ. Dresden, Inst. Analysis, Mommsenstr. 13, D - 01062 Dresden Supported by the DFG-Schwerpunkt-Programm "Dynamik: Analysis, effiziente Simulation und Ergodentheorie" 
maps of differential equations on manifolds. This is done in Section 3 of the paper. The differential equations are considered to be generated by time-dependent vector fields and the estimates are based on conditions for the eigenvalues of the symmetric part of the covariant derivative of the vector field.

'In the last Section 4 the number of closed orbits for autonomous differential equations is estimated from above in form of a Bendixson-Dulac criterion for Riemannian manifolds. For the derivation of the results properties of the Hausdorff measure as obtained in Section 2 play an essential role.

\section{Hausdorff dimension bounds for invariant sets of maps}

For a linear operator $T: E \rightarrow E^{\prime}$, where $E$ and $E^{\prime}$ are Euclidean spaces of dimension $n$, we write $T^{*}: E^{\prime} \rightarrow E$ for the adjoint operator of $T$. The singular values of $T$, i.e., the eigenvalues of the positive operator $\left(T^{*} T\right)^{1 / 2}: E \rightarrow E$, ordered with respect to its size and multiplicity we denote by $\alpha_{i}(T)(i=1, \ldots, n)$, i.e. $\alpha_{1}(T) \geq \alpha_{2}(T) \geq \ldots \geq \alpha_{n}(T)$.

For an arbitrary $k \in\{0,1, \ldots, n\}$ we define

$$
\omega_{k}(T)= \begin{cases}\alpha_{1}(T) \cdots \alpha_{k}(T) & \text { for } k>0 \\ 1 & \text { for } k=0 .\end{cases}
$$

More generally, for an arbitrary real number $d \in(0, n]$ written in the form $d=d_{0}+s$ with $d_{0} \in\{0,1, \ldots, n-1\}$ and $s \in(0,1]$ we introduce the notion

$$
\omega_{d}(T)=\omega_{d_{0}}(T)^{1-s} \cdot \omega_{d_{0}+1}(T)^{s}
$$

Obviously this can also be interpreted as

$$
\omega_{d}(T)=\left\|\wedge^{d_{0}} T\right\|^{1-s}\left\|\wedge^{d_{0}+1} T\right\|^{s}
$$

where $\left\|\wedge^{k} T\right\|$ stands for the norm of the $k$-exterior power of $T$, i.e. the norm of the linear operator $\wedge^{k} T: \wedge^{k} E \rightarrow \wedge^{k} E^{\prime}$.

Remark 2.1. Let $\Phi=\left(t_{j}^{i}\right)$ be the realization of the linear operator $T$ when fixing a basis $\left\{e_{i}\right\}$ in $E$ and a basis $\left\{e_{i}^{\prime}\right\}$ in $E^{\prime}$. Further, let us define the metric tensors by the matrices $G^{\prime}=\left(\left\langle e_{i}, e_{j}\right\rangle_{E}\right)$ and $G^{\prime}=\left(\left\langle e_{i}^{\prime}, e_{j}^{\prime}\right\rangle_{E^{\prime}}\right)$. The adjoint operator $T^{*}$ of $T$ is represented by the matrix $G^{-1} \Phi^{T} G^{\prime}$, where by $\Phi^{T}$ we mean the transposed matrix. Then the singular values are nothing else than the eigenvalues of the matrix $\sqrt{G^{\prime}} \Phi \sqrt{G^{-1}}$ or, what appears to be the same, they are the eigenvalues of the matrix $G^{-1} \Phi^{T} G^{\prime} \Phi$.

Before we pay attention to estimations of the Hausdorff dimension of compact negatively invariant with respect to a map sets on Riemannian manifolds we start with a short introduction of the notation of the Hausdorff dimension.

Let $(X, \rho)$ be a metric space and let $B_{\epsilon}(\xi)=\{\eta \in X \mid \rho(\xi, \eta) \leq \epsilon\}$ denote the closed ball in $X$ with center $\xi$ and radius $\epsilon$. For an arbitrary compact subset $K \subset X$ and for real numbers $d \geq 0$ and $\epsilon>0$ we consider the Hausdorff premeasure at level $\epsilon$ and of order $d$ of $K$.

$$
\mu(K, d, \epsilon)=\inf \sum_{i} r_{i}^{d}
$$


where the infimum is taken over all finite covers $\bigcup_{i} B\left(\xi_{i}, r_{i}\right) \supset K$ with balls of radius $r_{i} \leq \epsilon$. For fixed $d$ and $K$ the function $\mu(K, d, \epsilon)$ is monotone decreasing. Hence, the outer Hausdorff measure of order $d$ of $K$ can be defined as

$$
\mu(K, d)=\lim _{\epsilon \rightarrow+0} \mu(K, d, \epsilon)=\sup _{\epsilon>0} \mu(K, d, \epsilon) .
$$

Since the relation

$$
\mu(K, d+\delta, \epsilon) \leq \epsilon^{\delta} \mu(K, d, \epsilon)
$$

holds for every $d \geq 0, \epsilon>0$ and $\delta>0$, as can be easily verified, it turns out that there exists a unique number $d^{*}(K)$ with the property

$$
\mu(K, d)= \begin{cases}0 & \text { for } d>d^{*}(K) \\ +\infty & \text { for } d<d^{*}(K)\end{cases}
$$

This critical value $d_{H}(K):=d^{*}(K)$ is called Hausdorff dimension of $K$.

Let now $(M, g)$ be a Riemannian manifold without boundary of dimension $n$ and class $C^{1}$, let $U \subset M$ be an open subset and let us consider a map $\varphi: U \rightarrow M$ of class $C^{1}$. The tangent map of $\varphi$ at a point $u \in M$ is denoted by $d_{u} \varphi: T_{u} M \rightarrow T_{\varphi(u)} M$.

The following theorem generalizes the results of Douady and Oesterlé [4] for Riemannian manifolds.

Theorem 2.1. Let $d \in(0, n]$ be a real number and $K \subset U$ a compact set which is negatively invariant with respect to $\varphi$, i.e. $\varphi(K) \supset K$. If the inequality

$$
\sup _{u \in K} \omega_{d}\left(d_{u} \varphi\right)<1
$$

holds, then $d_{H}(K)<d$.

Remark 2.2. Let $u \in U$ be an arbitrary point and consider charts $x$ and $x^{\prime}$ at $u$ and $\varphi(u)$, respectively. We introduce the matrices $G=\left(g_{i j}(u)\right)$ and $G^{\prime}=\left(g_{i j}^{\prime}(\varphi(u))\right)$ that realize the metric fundamental tensor $g$ in the canonical bases of $T_{u} M$ and $T_{\varphi(u)} M$, respectively. The tangent map of $\varphi$ in $u$ written in coordinates of the charts $x$ and $x^{\prime}$ is then given by the matrix $\Phi=D\left(x^{\prime} \circ \varphi \circ x^{-1}\right)(x(u))$. From Remark 2.1 there follows that the singular values of the tangent map $d_{u} \varphi: T_{u} M \rightarrow T_{\varphi(u)} M$ coincide with the singular values of the matrix $\sqrt{G^{\prime}} \Phi \sqrt{G^{-1}}$.

The proof of Theorem 2.1 is postponed to the end of this section. Now we proceed with some corollaries. The first one concerns a result which has been formulated in $[2$, 8] for the case $M=\mathbb{R}^{n}$ using slightly stronger conditions for the map.

Corollary 2.1.1. Let $K \subset U$ be a compact set satisfying $\dot{\varphi}(K) \supset K$. If for some continuous function $p: U \rightarrow \mathbb{R}_{+}$and for some number $d \in(0, n]$ the inequality

$$
\sup _{u \in K} \omega_{d}\left(\frac{p(\varphi(u))}{p(u)} d_{u} \varphi\right)<1
$$


holds, then $d_{H}(K)<d$.

Proof. On the open set $U \subset M$ we, introduce a new metric tensor $\tilde{g}$ by

$$
\tilde{g}_{\left.\right|_{u}}=p^{2}(u) g_{\left.\right|_{\iota}} .
$$

It is easy to show that this is really a Riemannian metric equivalent to the given one on compact subsets of $M$. Since $K$ is compact the new equivalent metric does not alter the value of the Hausdorff dimension of $K$.

Let us now consider an arbitrary point $u \in K$ and two charts $x$ and $x^{\prime}$ around $u$ and $\varphi(u)$, respectively. Suppose that $G=\left(g_{i j}(u)\right)$ and $G^{\prime}=\left(g_{i j}^{\prime}(\varphi(u))\right)$ are the realizations with respect to the canonical bases in $T_{u} M$ and $T_{\varphi(u)} M$ of the metric tensors $g$ and $g^{\prime}$, respectively. As indicated in Remark 2.2 the singular values of the tangent map $d_{u} \varphi$ in the new metric are the singular values of the matrix

$$
\sqrt{\widetilde{G}^{\prime}} \Phi \sqrt{\widetilde{G}^{-1}}=p(\varphi(u)) \sqrt{G^{\prime}} \Phi p(u)^{-1} 1 \sqrt{G^{-1}}
$$

Thus, condition (2.4) guarantees that in the new metric the inequality (2.3) is valid and Theorem 2.1 can be applied

The next corollary provides a method for estimating the Hausdorff dimension without explicit computation of the singular values. It serves further as a basic tool to derive frequency domain conditions for feedback systems to receive upper bounds for the dimension [15].

Let us remark that for a linear operator $T: E \rightarrow \dot{E}^{\prime}$ where again $E$ and $E^{\prime}$ denote Euclidean spaces both of dimension $n$, the absolute value of the determinant is defined as $|\operatorname{det} T|=\left(\operatorname{det} T^{*} T\right)^{1 / 2}$.

Corollary 2.1.2. Let $K \subset U$ be a compact set which is assumed to be negatively invariant with respect to $\varphi$. Let $\theta: U \rightarrow \mathrm{R}_{+}$be a continuous function and let $d \in(0, n \mathrm{j}$ be a real number such that the conditions

(a) $\left\langle\left[\left(d_{u} \varphi\right)^{*} d_{u} \varphi\right] v, v\right\rangle \geq \theta(u)^{2}\|v\|^{2}$ for all $u \in K$ and $v \in T_{u} M$

(b) $\frac{\left|\operatorname{det} d_{u} \varphi\right|}{\theta(u)^{n-d}}<1$ for all $u \in K$

are satisfied. Then $d_{H}(K)<d$.

Proof. From condition (a) for the singular values $\alpha_{i}(u)$ of the tangent map $d_{u} \varphi$ we obtain the inequalities

$$
\alpha_{i}(u) \geq \theta(u) \quad \text { for all } u \in K \text { and } i=1,2, \ldots, n
$$

Thus, for any $k \in\{0,1, \ldots, n\}$ and $u \in K$ with $\alpha_{0}(u):=1$ it follows that

$$
\alpha_{1}(u) \cdots \alpha_{k}(u) \theta(u)^{n-k} \leq \alpha_{1}(u) \cdots \alpha_{n}(u)=\left|\operatorname{det} d_{u} \varphi\right|
$$


For $d=d_{0}+s$ with $d_{0} \in\{0,1, \ldots, n-1\}$ and $s \in(0,1]$ the last relation together with condition (b) of the corollary leads to

$$
\begin{aligned}
\alpha_{1}(u) \cdots \alpha_{d_{0}}(u) \alpha_{d_{0}+1}^{s}(u) & =\left(\alpha_{1}(u) \cdots \alpha_{d_{0}}(u)\right)^{1-s}\left(\alpha_{1}(u) \cdots \alpha_{d_{0}}(u) \alpha_{d_{0}+1}(u)\right)^{s} \\
& \leq \frac{\left|\operatorname{det} d_{u} \varphi\right|^{1-s}}{\theta(u)^{\left(n-d_{0}\right)(1-s)}} \frac{\left|\operatorname{det} d_{u} \varphi\right|^{s}}{\theta(u)^{\left(n-d_{0}-1\right) s}} \\
& =\frac{\left|\operatorname{det} d_{u} \varphi\right|}{\theta(u)^{n-d}} \\
& <1
\end{aligned}
$$

Now, the only thing left to do is to apply Theorem 2.1

For the proof of Theorem 2.1 we need some auxiliary results. Let $\mathcal{E}$ be an ellipsoid in an Euclidean space $E$ of dimension $n$ and let $\alpha_{1}(\mathcal{E}) \geq \ldots \geq \alpha_{n}(\mathcal{E})$ denote the lengths of the half-axes of $\mathcal{E}$ ordered with respect to its size. For an arbitrary real number $d \in(0, n]$ written in the form $d=d_{0}+s$ with $d_{0} \in\{0,1, \ldots, n-1\}$ and $s \in(0,1]$ the definition of the value $\omega_{d}(\mathcal{E})$ for $\mathcal{E}$ is provided by formula $(2.1)$ where $\alpha_{i}(T)$ is replaced by $\alpha_{i}(\mathcal{E})(i=1, \ldots, n)$.

In the first place we quote a result from [4] necessary for the proof of Theorem 2.1.

Lemma 2.1. Let us consider numbers $d \in(0, n]$ (written as above), $k>0, m>0$ and $\eta>0$ and assume $k \leq m^{d}$. Let $\mathcal{E}$ be an ellipsosd in $E$ such that $\alpha_{1}(\mathcal{E}) \leq m$ and $\omega_{d}(\mathcal{E}) \leq k$. Further we take a ball $B(0, \eta)$ of radius $\eta$ in $E$. Then the set $\mathcal{E}+B(0, \eta)$ is contained in an ellipsoid $\mathcal{E}^{\prime}$, which satisfies

$$
\omega_{d}\left(\mathcal{E}^{\prime}\right) \leq\left[1+\left(\frac{m^{d_{0}}}{k}\right)^{1 / s} \eta\right]^{d} k
$$

Let us come back now to the Riemannian manifold $(M, g)$ and consider the exponential map $\exp _{u}: T_{u} M \rightarrow M$ at an arbitrary point $u \in M$. Then the set $\exp _{u}(\mathcal{E})$ is the image of an ellipsoid $\mathcal{E}$ in the tangent space $T_{u} M$. centered at 0 under the map exp . $_{u}$. We recall the definition of another outer measure concerning covers of such images of ellipsoids in tangent space and then are interested in the relation between the outer Hausdorff measure and this ellipsoid measure.

Let $K \subset U$ be a compact set, let $\epsilon>0$ be a sufficiently small number and fix a number $d \in(0, n]$. The outer ellipsoid premeasure at level $\epsilon$ and of order $d$ of $K$ is given by

$$
\tilde{\mu}(K, d, \epsilon)=\inf \sum_{i} \omega_{d}\left(\mathcal{E}_{i}\right)
$$

with infimum is taken over all finite covers $\bigcup_{i} \exp _{u_{i}}\left(\mathcal{E}_{i}\right) \supset K$, where $u_{i} \in M$ and $\mathcal{E}_{i} \in T_{u_{i}} M$ are ellipsoids satisfying $\omega_{d}\left(\mathcal{E}_{i}\right)^{1 / d} \leq \epsilon$. Now we show the equivalence of both the Hausdorff premeasure and the ellipsoid premeasure in a similar way as it is done in [4]. 
Lemma 2.2. For an arbitrary number $d \in(0, n]$ written in the form $d=d_{0}+s$ with $d_{0} \in\{0,1, \ldots, n-1\}$ and $s \in(0,1]$ we define the numbers $C_{d}=2^{d_{0}}\left(d_{0}+1\right)^{d / 2_{i}}$ anid $\lambda_{d}=\sqrt{d_{0}+1}$. Then for a compact set $K \subset U$ and for every sufficiently small $\epsilon>0$ the inequality

$$
\mu(K, \dot{d}, \epsilon) \geq \tilde{\mu}(K, d, \epsilon) \geq C_{d}^{-1} \mu\left(K, d, \dot{\lambda_{d}} \epsilon\right)
$$

holds.

Proof. In a number of technical details the proof differs from the one given in [4]. Therefore, at least in short we show some of the essential steps.

In analogous manner as in [4] it is established that for sufficiently small $\epsilon>0$, for an arbitrary $u \in K$ and any ellipsoid $\mathcal{E} \subset T_{u} M$ satisfying $\omega_{d}(\mathcal{E})^{1 / d} \leq \epsilon$ the relation

$$
\mu\left(\exp _{u}(\mathcal{E}), d, \lambda_{d} \epsilon\right) \leq C_{d} \omega_{d}(\mathcal{E})
$$

is valid. Let us now fix a finite cover of $K$ consisting of sets $\left\{\exp _{u_{i}}\left(\mathcal{E}_{i}\right)\right\}_{i}$, where $\omega_{d}\left(\mathcal{E}_{i}\right)^{1 / d} \leq \epsilon$ holds for all indices $i$. The properties of the measure then guarantee the relation

$$
\left.\mu\left(K, d, \lambda_{d} \epsilon\right) \leq \mu\left(\bigcup_{i} \exp _{u_{i}}\left(\mathcal{E}_{i}\right), d, \lambda_{d} \epsilon\right) \leq \sum_{i} \mu\left(\exp _{u_{i}} \mathcal{E}\right), d, \lambda_{d} \epsilon\right)
$$

Using (2.6) we obtain $\mu\left(K, d, \lambda_{d} \epsilon\right) \leq C_{d} \sum_{i} \omega_{d}\left(\mathcal{E}_{i}\right)$ and since the cover was arbitrary among all those satisfying the restriction for $\omega_{d}$ we have $\mu\left(K, d, \lambda_{d} \epsilon\right) \leq C_{d} \widetilde{\mu}(K, \dot{d}, \epsilon)$

Lemma 2.3. Let $K \subset U$ be a compact set and consider a map $\varphi: U \rightarrow \dot{M}$ of class $C^{1}$. For a number $d \in(0, n]$ we assume that $\sup _{u \in K} \omega_{d}\left(d_{u} \varphi\right) \leq k$. Then, for every $l>k$ there exists a number $\epsilon_{0}>0$ such that for every $\epsilon \in\left(0, \epsilon_{0}\right]$

$$
\mu\left(\varphi(K), d, \lambda_{d} l^{1 / d} \epsilon\right) \leq C_{d} l \mu(K, d, \epsilon)
$$

holds, where $C_{d}$ and $\lambda_{d}$ are define $\ddot{d}$ as in Lemma 2.2 .

Proof. In a first step we show for sufficiently small numbers $\epsilon>0$ the inequality

$$
\widetilde{\mu}\left(\varphi(K), d, l^{1 / d} \epsilon\right) \leq l \mu(\ddot{k}, d, \epsilon)
$$

Obviously it is always possible to find an open set $V \subset U$ containing $K$ which itself lies inside a compact set $\widetilde{K} \subset U$ with the property

$$
k^{\prime}:=\sup _{u \in \widetilde{K}} \omega_{d}\left(d_{u} \varphi\right)<l
$$

We choose a number $\dot{m}>0$ such that $k^{\prime}<\dot{m}^{d}$ and

$$
\sup _{u \in \widetilde{K}}\left\|d_{u} \varphi\right\| \leq m
$$


hold. Further we can find a number $\eta>0$ satisfying

$$
\left[1+\left(\frac{m^{d_{0}}}{k^{\prime}}\right)^{1 / s} \eta\right]^{d} k^{\prime} \stackrel{=}{=} .
$$

We take $\epsilon>0$ small enough such that

$$
\epsilon \leq \frac{1}{2} \inf _{\substack{u \in K \\ u^{\prime} \in U \backslash v}} \rho\left(\dot{u}, u^{\prime}\right) \text { and }\left\|\tau_{\varphi(v)}^{\varphi(u)} d_{v} \varphi \tau_{u}^{v}-d_{u} \varphi\right\| \leq \eta
$$

for: all $u ; v, \in V$ with $\rho(u, v) \leq \epsilon . \cdot \mathrm{By}: \rho(\cdot, \cdot)$ we mean the geodesic distance between the points of $M$ and by $\tau_{u}^{v}$ we denote the isometry between $T_{u} M$ and $T_{v} M$ 'defined by parallel transport along the geodesic for points lying sufficiently near to each other.

Let us fix a finite cover with.balls $\left\{B\left(u_{i}, r_{i}\right)\right\}_{i}$ of radius $r_{i} \leq \cdot \epsilon$ of $K$. Then every ball $B\left(u_{i}, r_{i}\right)$ satisfying $B\left(u_{i}, r_{i}\right) \cap K \neq \emptyset$ is entirely contained in the open set $V$. The Taylor formula for differentiable maps provides that for every $v \in B\left(u_{i}, r_{i}\right)$.

$$
\begin{aligned}
\left\|\exp _{\varphi\left(u_{i}\right)}^{-1} \varphi(v)-d_{u_{i}} \varphi\left(\exp _{u_{i}}^{-1}(v)\right)\right\| & \vdots \\
& \leq \sup _{w \in B\left(u_{i}, r_{i}\right)}\left\|\tau_{\varphi(w)}^{\varphi\left(u_{i}\right)} d_{w} \varphi \tau_{u_{i}}^{w}-d_{u_{i}} \varphi\right\| \cdot\left\|\exp _{u_{i}}^{-1}(w)\right\|
\end{aligned}
$$

holds. Thus, for every ball $B\left(u_{i}, r_{i}\right)$ of the cover with $B\left(u_{i}, r_{i}\right) \cap K \neq \emptyset$ for the image under the map $\varphi$ we have the inclusion

$$
\varphi\left(B\left(u_{i}, r_{i}\right)\right) \subset \exp _{\varphi\left(u_{i}\right)}\left(d_{u_{i}} \varphi\left(B_{T_{u_{i}} M}\left(0, r_{i}\right)\right)+B_{T_{\varphi\left(u_{i}\right)} M}\left(0, \eta r_{i}\right)\right)
$$

The notions $B_{T_{u_{i}} M}\left(0, r_{i}\right)$ and $B_{T_{\varphi\left(u_{i}\right)} M}\left(0, \eta r_{i}\right)$ stand for balls in the tangent spaces $T_{u_{i}} M$ and $T_{\varphi\left(u_{i}\right)} M$, respectively. Obviously the set $d_{u_{i}} \varphi\left(B_{T_{u_{i}} M}\left(0, r_{i}\right)\right)$ is an ellipsoid with half-axes of length $r_{i} \alpha_{j}\left(u_{i}\right)$, where $\alpha_{j}\left(u_{i}\right)$ denote the singular values of the linear operator $d_{u_{i}} \varphi \quad(i=1, \ldots, n)$. Concerning the definition of $k^{\prime}$ we may conclude :

$$
\omega_{d}\left(d_{u_{i}} \varphi\left(B_{T_{u_{i}} M}\left(0, r_{i}\right)\right)\right) \leq r_{i}^{d} k^{\prime} .
$$

With (2.8). it follows

$$
\alpha_{1}\left(d_{u_{i}} \varphi\left(B_{T_{u_{i}} M}\left(0 ; r_{i}\right)\right)\right) \leq m r_{i}
$$

Further by using (2.9), (2.12) and (2.13) and by Lemma 2.1 there can be found an ellipsoid $\mathcal{E}_{i}^{\prime}$ containing $\exp _{u_{i}}^{-1}\left(\varphi\left(B\left(u_{i}, r_{i}\right)\right)\right)$ and satisfying $\omega_{d}\left(\mathcal{E}_{i}^{\prime}\right) \leq l r_{i}^{d}$.

We can resume that every finite cover of the compact set $K$ with balls $\left\{B\left(u_{i}, r_{i}\right)\right\}_{i}$ of radius $r_{i} \leq \epsilon$ such that $B\left(u_{i}, r_{i}\right) \cap K$ is non-empty generates a cover $\left\{\exp _{u_{i}}\left(\mathcal{E}_{i}^{\prime}\right)\right\}_{i}$ of $\varphi(K)$, where $\mathcal{E}_{i}^{\prime}$ denotes an ellipsoid in $T_{u_{i}} M$ satisfying $\omega_{d}\left(\mathcal{E}_{i}^{\prime}\right) \leq l r_{i}^{d}$. Therefore, we have

$$
\sum_{i} \omega_{d}\left(\mathcal{E}_{i}^{\prime}\right) \leq l \sum_{i} r_{i}^{d}
$$


Since the result is valid for every such cover it must be true for the infimum as.well. So we have

$$
\sum_{i} \omega_{d}\left(\mathcal{E}_{i}^{\prime}\right) \leq l \mu(k, d, \epsilon)
$$

If at the left-hand side we pass to the infimum, then the last inequality becomes (2.7). Lemma 2.2 and (2.7) finally guarantee the inequalities

$$
l \mu(K, d, \epsilon) \geq \tilde{\mu}\left(\varphi(K), d, l^{1 / d} \epsilon\right) \geq C_{d}^{-1} \mu\left(\varphi(K), d, \lambda_{d} l^{1 / d} \epsilon\right) .
$$

But this is exactly what we wanted to proof

Proof of Theorem 2.1. The essence of the proof of Theorem 2.1 is contributed by Lemma 2.3. The lemma claims that the Hausdorff premeasure defined on a Riemannian manifold exhibits the same properties concerning the effect of a map $\varphi$ of class $C^{1}$ as it has in $\mathrm{R}^{n}$. When applying Lemma 2.3 the statement of Theorem 2.1 follows directly from arguments that agree with the last steps of the proof of Theorem 1 in [4]

The next theorem provides another important result the proof of which follows directly from Lemma 2.3 using the same arguments as in [2].

Theorem 2.2. Let the manifold $M$ be compact and let $\varphi: M \rightarrow M$ be a map of class $C^{1}$. Suppose that $\sup _{u \in M} \omega_{d}\left(d_{u} \varphi\right)<1$ holds for a number $d \in(0, n]$. If for a compact set $K \subset M$ the condition $\mu(K, d)<\infty$ is satisfied, then

$$
\lim _{m \rightarrow \infty} \mu\left(\varphi^{m}(K), d\right)=0
$$

\section{Time-dependent vector fields}

Let $(M, g)$ be a Riemannian manifold without boundary of dimension $n$ and of class $C^{2}$, let $U \subset M$ be an open subset and $I_{1} \subset \mathrm{R}$ an open interval with $0 \in I_{1}$. We consider a time-dependent vector field $f: I_{\mathrm{i}} \times U \rightarrow T U$ of class $C^{1}$ and the corresponding differential equation

$$
\dot{u}=f(t, u) .
$$

Suppose that for a point $(t, u) \in I_{1} \times U$ the covariant derivative of the vector field $f$ is $\nabla f(t, u): T_{u} M \rightarrow T_{u} M$. We assume for (3.1) that there can be found an open set $D \subset U$ and an open interval $I \subset I_{1}$ such that the solution $\varphi(\cdot, u)$ of equation (3.1) starting at $u \in D$ for $t=0$ exists everywhere on $I$. For every $t \in I$ we define the $t$-shift operator $\varphi^{t}: D \rightarrow U$ by $\varphi^{t}(u)=\varphi(t, u)$. In case the differential equation (3.1) is autonomous the family $\left\{\varphi^{t}\right\}_{t \in I}$ of all those $t$-shifts is a local flow.

Since the vector field $f$ is continuously differentiable the same holds for the $t$-shift operators $\varphi^{t}(t \in I)$. For an arbitrary point $u \in D$ the tangent map $d_{u} \varphi^{t}$ solves the variation equation

$$
y^{\prime}=\nabla f\left(t, \varphi^{t}(u)\right) y
$$

with initial condition $d_{u} \varphi_{\mid t=0}^{t}=\mathrm{id}_{T_{u} M}$. Here the absolute derivative $y^{\prime}$ is taken along the integral curve $t \mapsto \varphi^{t}(u)$ in the direction of the vector field $f$. Let us denote 
the eigenvalues of the symmetric part of the covariant derivative $\nabla f(t, u)$, i.e. of the operator

$$
S(t, u)=\frac{1}{2}\left[\nabla f(t, u)+\nabla f(t, u)^{*}\right]
$$

by $\lambda_{i}(t, u) \quad(i=1, \ldots, n)$ and order them with respect to its size and multiplicity, i.e. $\lambda_{1}(t, u) \geq \ldots \geq \lambda_{n}(t, u)$. The divergence $\operatorname{div} f(t, u)$ of the vector field $f$ at $(t, u) \in I_{1} \times U$ is the trace of the linear operator $\nabla f(t, u): T_{u} M \rightarrow T_{u} M$ and therefore

$$
\operatorname{div} f(t, u)=\operatorname{tr} \nabla f(t, u)=\lambda_{1}(t, u)+\ldots+\lambda_{n}(t, u)
$$

holds.

The next theorem is the main result of this section and extends a result of [17] to Riemannian manifolds. The proof is given at the end of the section.

Theorem 3.1. Let $d \in(0, n]$ be a real number written in the form $d=d_{0}+s$ with $d_{0} \in\{0,1, \ldots, n-1\}$ and $s \in(0,1]$ and let $K \subset D$ be a compact set satisfying $\varphi^{\tau}(K) \supset K$ for a certain $\tau \in I \cap \mathrm{R}_{+}$. If the condition

$$
\sup _{u \in K} \int_{0}^{T}\left[\lambda_{1}\left(t, \varphi^{t}(u)\right)+\ldots+\lambda_{d_{0}}\left(t, \varphi^{t}(u)\right)+s \lambda_{d_{0}+1}\left(t, \varphi^{t}(u)\right)\right] d t<0
$$

holds, then $d_{H}(K)<d$.

Remark 3.1. We consider an arbitrary $u \in U$ and a chart $x$ around $u$. In local coordinates of $x$ and in the canonical basis $\partial_{1}(u), \ldots, \partial_{n}(u)$ of the tangent space $T_{u} M$ the vector field of (3.1) then becomes $f(t, u)=f^{i}(t, u) \partial_{i}(u)$ and the covariant derivative $\nabla f(t, u): T_{u} M \rightarrow T_{u} M: v \mapsto \nabla_{v} f(t, u)$ is given by $\nabla_{v} f(t, u)=\dot{\nabla}_{i} f^{k}(t, u) v^{i} \partial_{k}(u)$, where $v=v^{i} \partial_{i}(u)$ is an arbitrary vector in $T_{u} M$ and

$$
\nabla_{i} f^{k}=\frac{\partial f^{k}}{\partial x^{i}}+\Gamma_{i j}^{k} f^{j}
$$

Here by $\Gamma_{i j}^{k}$ the Christoffel symbols in the chart $x$ corresponding to the metric tensor $g$ are denoted. The symmetric part $S(t, u)$ of $\nabla f(t, u)$ in the canonical basis of $T_{u} M$ is realized by the matrix

$$
\frac{1}{2}\left[G^{-1} \Phi^{T} G+\Phi\right]
$$

where $G$ is as in Remark 2.2 and $\Phi=\left(\nabla_{i} f^{k}\right)$. The expression for the variation equation (3.2). in the chart $x$ is

$$
y^{k i}=\dot{y}^{k}+\Gamma_{i j}^{k} f^{j} y^{i}=\nabla_{i} f^{k} y^{i}
$$

Let us define $f_{s, i}=g_{s i} \nabla_{i} f^{t}$ and consider the quadratic form $e_{s i}=\frac{1}{2}\left[f_{s, i}+f_{i, s}\right]$. Then $e_{s i}$ is related to (3.4) in the sense that the eigenvalues of this quadratic form, i.e. the solutions of $\operatorname{det}\left[e_{s i}-\lambda g_{s i}\right]=0$ coincide with the eigenvalues of the matrix (3.4). Let us now by means of the notion $c_{j k}=g_{i k} \Gamma_{j,}^{i} f^{s}$ introduce the derivative of the metric tensor 
$g$ in the direction of the vector field $f^{i}$ by $\dot{g}_{j k}=\frac{1}{2}\left[c_{j k}+c_{k j}\right]$. Then the quadratic form can be written as

$$
e_{s i}=\frac{1}{2}\left[g_{s k} \frac{\partial f^{k}}{\partial x^{i}}+\frac{\partial f^{k}}{\partial x^{s}} g_{i k}\right]+\dot{g}_{s i}
$$

Before we devote ourselves to the proof of Theorem 3.1 we add some corollaries. The first one generalizes a result of $[2,8]$ formulated there for the case $M=\mathbb{R}^{n}$ and under slightly stronger conditions. Keeping in mind the second method of Lyapunov which is often used in stability theory that result is in $[2,8]$ referred to as "introduction of a Lyapunov function in Hausdorff dimension estimates". In our point of view that approach can be treated as an introduction of a new metric tensor on the manifold.

For a differentiable function $v: U \rightarrow \mathrm{R}$ the map $\dot{v}: I_{1} \times U \rightarrow \mathrm{R}$ defined by $\dot{v}(t, u)=d_{u} v \cdot f(t, u)$ is the derivative of $v$ in the direction of the vector field $f$.

Corollary 3.1.1 Let $K \subset D$ be a compact set such that $\varphi^{\top}(K) \supset K$ is true for some $\tau \in I \cap \mathrm{R}_{+}$. Let $v: U \rightarrow \mathrm{R}$ be a differentiable function and denote by $\lambda_{1}(t, u) \geq$ $\ldots \geq \lambda_{n}(t, u)$ the eigenvalues of $S(t, u)$. If for a real number $d \in(0, n]$ written in the form $d=d_{0}+s$ with $d_{0} \in\{0,1, \ldots, n-1\}$ and $s \in(0,1]$ the condition

$$
\begin{gathered}
\sup _{u \in K} \int_{0}^{\tau}\left[\lambda_{1}\left(t, \varphi^{t}(u)\right)+\cdots+\lambda_{d_{0}}\left(t, \varphi^{t}(u)\right)\right. \\
\left.\quad+s \lambda_{d_{0}+1}\left(t, \varphi^{t}(u)\right)+\dot{v}\left(t, \varphi^{t}(u)\right)\right] d t \\
<0
\end{gathered}
$$

holds, then $d_{H}(K)<d$.

Proof. We introduce on $\mathrm{U}$ a new metric tensor $\tilde{g}_{\left.\right|_{u}}=p^{2}(u) g_{\left.\right|_{u}}$ by means of some function $p: U \rightarrow \mathbb{R}_{+}$of class $C^{1}$. Let us fix a point $u \in U$ and consider a chart $x$ around $u$. Further, let the metric tensor $g$ and the vector field $f$ be expressed in the canonical basis of $T_{u} M$ by $g_{i j}$ and $f^{i}$, respectively. The symmetric part of the covariant derivative $\widetilde{\nabla} f(t, u)$ at $u \in U$ with respect to the new metric is then determined according to Remark 3:1 by the matrix representation

$$
\frac{1}{2}\left[G^{-1} \Phi^{T} G+\Phi\right]+\frac{\dot{p}}{p} \text { Id }
$$

If, in particular, we choose $p(u)=e^{\frac{v(u)}{d}}(u \in U)$, then $\dot{p}(t, u)=p(u) \frac{\dot{v}(u)}{d}$ implies that the eigenvalues $\tilde{\lambda}_{i}$ of (3.6) are related to the eigenvalues with respect to the original metric $g$ by the formula $\tilde{\lambda}_{i}=\lambda_{i}+\frac{\dot{v}}{d}$. Finally

$$
\tilde{\lambda}_{1}+\cdots+\tilde{\lambda}_{d_{0}}+s \tilde{\lambda}_{d_{0}+1}=\lambda_{1}+\cdots+\lambda_{d_{0}}+s \lambda_{d_{0}+1}+\dot{v}
$$

guarantees (3.5) and therefore (3.3) of Theorem 3.1 
To verify the conditions of Theorem 3.1 we need to compute the eigenvalues of the symmetric part of the covariant derivative. The next two corollaries are variations of this theorem using conditions on the divergence of the vector field of (3.1). Similar results for the case $M=\mathbb{R}^{n}$ can be found in [17]. Unlike [17] the proof given below follows directly from Theorem 3.1 . In $[2,8,17]$ these results form a part of statements which are fundamental for an effective realization of dimension estimates by using the solvability of special matrix equations.

Corollary 3.1.2. Let $K \subset D$ be a compact set such that $\varphi^{\top}(K) \supset K$ holds for some $\tau \in I \cap \mathbb{R}_{+}$. Assume that for a continuous function $\theta: I \times U \rightarrow \mathbb{R}$ and for some $d \in(0, n]$ the conditions

(a) $\langle S(t, u) v, v\rangle \geq-\theta(t, u)\|v\|^{2}$ for all $t \in[0, \tau], u \in U$ and $v \in T_{u} M$

(b) $\sup _{u \in K} \int_{0}^{r}\left[\operatorname{div} f\left(t, \varphi^{t}(u)\right)+(n-d) \theta\left(t, \varphi^{t}(u)\right)\right] d t<0$

are satisfied. Then $d_{H}(K)<d$.

Proof. From condition (a) we have for the eigenvalues $\lambda_{i}$ of $S(t, u)$

$$
\lambda_{i}(t, u) \geq-\theta(t, u) \quad \text { for all }(t, u) \in[0, \tau] \times U \text { and } i=1, \ldots, n \text {. }
$$

Thus, if $k \in\{0,1, \ldots, n\}, t \in[0, \tau], u \in U$ and $\lambda_{0}(t, u)=0$ are arbitrary, then

$$
\lambda_{1}(t, u)+\cdots+\lambda_{k}(t, u)-(n-k) \theta(t, u) \leq \operatorname{tr} S(t, u)=\operatorname{div} f(t, u) .
$$

This implies

$$
\lambda_{1}(t, u)+\cdots+\lambda_{d_{0}}(t, u)+s \lambda_{d_{0}+1}(t, u) \leq \operatorname{div} f(t, u)+(n-d) \theta(t, u) .
$$

By using condition (b) and Theorem 3.1 the proof is complete

Corollary 3.1.3. Consider (3.1) on an open set $U \subset \mathbb{R}^{n}$. Suppose that system (3.1) possesses a compact set $K$ satisfying $\varphi^{\top}(K) \supset K$ for some $\tau \in I \cap \mathbb{R}_{+}$. Further, assume that there exist a number $d \in(0, n]$, an $(n \times n)$-Matrix $H=H^{T} .>0$ and a continuous function $\theta: I \times U \rightarrow \mathbb{R}$ such that the condition (b) of Corollary 9.1.2 and the inequality

$$
\frac{1}{2}\left[H D_{2} f(t, u)+D_{2} f(t, u)^{T} H\right] \geq-\theta(t, u) H \quad \text { for all }(t, u) \in[0, \tau] \times U
$$

are satisfied. Then $d_{H}(K)<d$.

Proof. We introduce in $U$ a new metric by means of the matrix $\left(g_{i j}\right) \equiv H$. From Remark 3.1 it follows that the eigenvalues $\lambda_{i}(t, u)$ of $S(t, u)$ with respect to the new metric agree with the eigenvalues of the quadratic form corresponding to (3.4). Therefore, they satisfy the relation

$$
\frac{1}{2}\left[H D_{2} f(t, u)+D_{2} f(t, u)^{T} H\right]=\lambda_{i}(t, u) H .
$$

Using this and (3.8) we obtain (3.7). All further steps can analogously be done as in the proof of Corollary 3.1.2 
In the proof of Theorem 3.1 given below several properties of linear operators in exterior products of Euclidean spaces $[5,6,18]$ are used. Let $E$ be an Euclidean space of dimension $n$ and let $T: E \rightarrow E$ be a linear operator. For any number $k \in\{1, \ldots, n\}$ the $k$-th derivation operator $T_{k}: \bigwedge^{k} E \rightarrow \bigwedge^{k} E$ is defined by

$$
T_{k}=\frac{d}{d s}\left[\bigwedge^{k}\left(\mathrm{id}_{E}+s T\right)\right]_{\mid s=0} .
$$

For any points $\xi_{1}, \ldots, \xi_{k} \in E$ this operator satisfies the equation

$$
\begin{aligned}
T_{k}\left(\xi_{1} \wedge \ldots \wedge \xi_{k}\right)= & T \xi_{1} \wedge \xi_{2} \wedge \ldots \wedge \xi_{k} \\
& +\xi_{1} \wedge T \xi_{2} \wedge \ldots \wedge \xi_{k}+\ldots+\xi_{1} \wedge \xi_{2} \wedge \ldots \wedge T \xi_{k} .
\end{aligned}
$$

If $\lambda_{1}(T), \ldots, \lambda_{n}(T)$ denotes the complete system of eigenvalues of the operator $T$, then the corresponding complete system of eigenvalues of the operator $T_{k}$ consists of the $\left(\begin{array}{l}n \\ k\end{array}\right)$ numbers $\lambda_{i_{1}}(T)+\ldots+\lambda_{i_{k}}(T)$ with indices $1 \leq i_{1}<\ldots<i_{k} \leq n$ (see, for example, [11]). In case $T$ is selfadjoint all the eigenvalues of $T$ are real and can be ordered in the way $\lambda_{1}(T) \geq \ldots \geq \lambda_{n}(T)$. The operator $T_{k}$ is then also selfadjoint and it follows

$$
\begin{aligned}
& \lambda_{\min }\left(T_{k}\right)=\lambda_{n-k+1}(T)+\ldots+\lambda_{n}(T) \\
& \lambda_{\max }\left(T_{k}\right)=\lambda_{1}(T)+\ldots+\lambda_{k}(T) .
\end{aligned}
$$

Proof of Theorem 3.1. Let us fix an arbitrary $u \in K$, a number $k \in\{1, \ldots, n\}$ and arbitrary $v_{1}, \ldots, v_{k} \in T_{u} M$. For every $t \in[0, \tau]$ we introduce

$$
w(t)=\left\|d_{u} \varphi^{t} v_{1} \wedge \ldots \wedge d_{u} \varphi^{t} v_{k}\right\|_{\wedge^{k} T_{\varphi^{t}(u)} M}^{2} .
$$

Applying the variation equation (3.2) and formula (3.9) we achieve for every $t$ in $[0, \tau]$ the equation

$$
\dot{w}(t)=2\left\langle\left[S\left(t, \varphi^{t}(u)\right)\right]_{k}\left(d_{u} \varphi^{t} v_{1} \wedge \ldots \wedge d_{u} \varphi^{t} v_{k}\right), d_{u} \varphi^{t} v_{1} \wedge \ldots \wedge d_{u} \varphi^{t} v_{k}\right\rangle_{\wedge^{k} T_{\varphi^{t}(u)} M}
$$

With (3.10) for every $t \in[0, \tau]$ this leads to

$$
\begin{aligned}
2\left[\lambda_{n-k+1}\right. & \left.\left(t, \varphi^{t}(u)\right)+\ldots+\lambda_{n}\left(t, \varphi^{t}(u)\right)\right] w(t) \\
& \leq \dot{w}(t) \leq 2\left[\lambda_{1}\left(t, \varphi^{t}(u)\right)+\ldots+\lambda_{k}\left(t, \varphi^{t}(u)\right)\right] w(t)
\end{aligned}
$$

Therefore we conclude

$$
\begin{aligned}
\| d_{u} \varphi^{\top} v_{1} & \wedge \ldots \wedge d_{u} \varphi^{\top} v_{k} \|_{\wedge^{k} T_{\varphi^{t}(u)} M} \\
\leq & \left\|v_{1} \wedge \ldots \wedge v_{k}\right\|_{\wedge^{k} T_{u} M} \\
& \times \exp \left\{\int_{0}^{\tau}\left[\lambda_{1}\left(t, \varphi^{t}(u)\right)+\ldots+\lambda_{k}\left(t, \varphi^{t}(u)\right)\right] d t\right\} .
\end{aligned}
$$


We apply the Courant-Hilbert-Fischer Theorem to the product of the squares of the first $k$ singular values of $d_{u} \varphi^{r}$ and use (3.12) in order to receive that

$$
\begin{aligned}
& \alpha_{1}^{2}(\tau, u) \cdots \alpha_{k}^{2}(\tau, u)=\lambda_{1}\left(\bigwedge^{k}\left[\left(d_{u} \varphi^{r}\right)^{*} d_{u} \varphi^{r}\right]\right) \\
& =\sup _{\substack{u \in \Lambda^{k} T_{u} M \\
\|\|_{\Lambda^{k} T_{u} M^{\prime}=1}}}\left\|\Lambda^{k} d_{u} \varphi^{\tau} v\right\|_{\Lambda^{k} T_{\varphi^{\top}(u)} M}^{2} \\
& =\sup _{\substack{v_{1}, \ldots, v_{k} \in T_{u} M \\
\left\|u_{i}\right\| T_{u} M=1}}\left\|d_{u} \varphi^{T} v_{1} \wedge \ldots \wedge d_{u} \varphi^{T} v_{k}\right\|_{\wedge^{k} T_{\varphi} T_{(u)} M}^{2} \\
& \leq \exp \left\{2 \int_{0}^{\tau}\left[\lambda_{1}\left(t, \varphi^{t}(u)\right)+\cdots+\lambda_{k}\left(t, \varphi_{:}^{t}(u)\right)\right] d t\right\} .
\end{aligned}
$$

This last inequality and the assumptions of Theorem 3.1 finally guarantee

$$
\begin{aligned}
& \sup _{u \in K} \omega_{d}\left(d_{u} \varphi^{r}\right) \\
& \quad=\sup _{u \in K}\left[\alpha_{1}(\tau, u) \cdots \alpha_{d_{0}}(\tau, u)\right]^{1-s}\left[\alpha_{1}(\tau, u) \cdots \alpha_{d_{0}+1}(\tau, u)\right]^{s} \\
& \leq \sup _{u \in K} \exp \left\{\int_{0}^{\tau}\left[\lambda_{1}\left(t, \varphi^{t}(u)\right)+\cdots+\lambda_{d_{0}}\left(t, \varphi^{t}(u)\right)+s \lambda_{d_{0}+1}\left(t, \varphi^{t}(u)\right)\right] d t\right\} \\
& <1 .
\end{aligned}
$$

This shows that for the map $\varphi^{r}$ the assumptions of Theorem 2.1 are valid

Remark 3.2. The inequalities (3.11) can be interpreted as a generalization of Liouville's truncated trace formula for linear differential equations in Euclidean spaces [17]. In particular from (3.11) by setting $k=n$ and indicating that

$$
\lambda_{1}\left(t, \varphi^{t}(u)\right)+\ldots+\lambda_{n}\left(t, \varphi^{t}(u)\right)=\operatorname{tr} \nabla f\left(t, \varphi^{t}(u)\right)=\operatorname{div} f\left(t, \varphi^{t}(u)\right)
$$

we obtain Liouville's trace formula in the form

$$
\left(\left|\operatorname{det} d_{u} \varphi t\right|\right)^{\circ}=\operatorname{div} f\left(t, \varphi^{t}(u)\right)\left|\operatorname{det} d_{u} \varphi t\right| \quad \text { for all } t \in[0, \tau] \text { and } u \in K \text {. }
$$

For a point $u$ and a chart $x$ in the neighbourhood of this point let again $g_{i j}, f^{i}$ and $\xi^{i}$ represent $g, f$ and $\varphi^{t}$ in coordinates of the chart $x$. Then formula (3.15) agrees locally with

$$
\left(\sqrt{\gamma}\left|\operatorname{det}\left(\frac{\partial \xi^{i}}{\partial x^{j}}\right)\right|\right)=\nabla_{k} \xi^{k} \sqrt{\gamma}\left|\operatorname{det}\left(\frac{\partial \xi^{i}}{\partial x^{j}}\right)\right| .
$$

where $\gamma$ stands for $\operatorname{det}\left(g_{i j}\right)$.

For a Lebesgue measurable set $\Omega \subset D$ of finite volume we denote the volume of $\varphi^{t}(\Omega)$ by $V_{t}$. Then the formula (3.15) provides the transport lemma [1] for Riemannian manifolds in the form

$$
\dot{V}_{t}=\int_{\varphi^{t}(\Omega)} \operatorname{div} f d V
$$




\section{Convergence for autonomous vector fields}

We now consider compact Riemannian manifolds $(M, g)$ without boundary of dimension $n$ and of class $C^{2}$. Let on $M$ be given a vector field $f: M \rightarrow T M$ of class $C^{1}$ and the corresponding differential equation

$$
\dot{u}=f(u) \text {. }
$$

We assume that the global flow $\varphi: \mathrm{R} \times M \rightarrow M$ of (4.1) exists. As in the previous section we denote by $\lambda_{1}(u) \geq \ldots \geq \lambda_{n}(u)$ the eigenvalues of the symmetric part $S(u)=$ $\frac{1}{2}\left[\nabla f(u)^{*}+\nabla f(u)\right]$ of the covariant derivative $\nabla f(u): T_{u} M \rightarrow T_{u} M$ of $f$ at a point $u \in M$.

The main result of this section is Theorem 4.1 which can be considered as a general formulation of the Bendixson-Dulac criterion for Riemannian manifolds of dimension $n$. Certain generalizations of the original Bendixson-Dulac criterion for differential equations in $R^{n}$ (see $[2,3,17]$ or for vector fields on the flat cylinder $[9,10]$ ) can be derived from that theorem when adapting it to the particular situation.

In the following the dimension of the 1-homology group $H_{1}(M)$ of $M$ is denoted by $b_{1}$, i.e. the first Betti number of $M$.

Theorem 4.1. Let the manifold $M$ with Betti number $b_{1}$ be compact and suppose that for the eigenvalues of the symmetric part $S$ of $\nabla f$ one of the inequalities.

(a) $\lambda_{1}(u)+\lambda_{2}(u)<0$

(b) $\lambda_{n-1}(u)+\lambda_{n}(u)>0$

is valid on $M$. Then the system (4.1) possesses on $M$ at most $b_{1}$ non-trivial periodic orbits.

Proof. We only consider the case of condition (a), the other one can be performed in a similar fashion by considering the negative time evolution of the flow of equation (4.1). We first take $b_{1}=0$ and show that every closed orbit is constant. Suppose that (4.1) has a nontrivial closed orbit $\gamma$. Let $S$ be a surface (membrane) in $M$ of minimal two-dimensional Hausdorff measure $0<\mu(S, 2)<\infty$ with boundary $\gamma$. Notice, that such a surface as solution of the Plateau problem for Riemannian manifolds $[6,13]$ in our situation exists. The properties of the flow ensure that for arbitrary $t \geq 0$ the set $\varphi^{t}(S)$ is again a membrane in $M$ with boundary $\gamma$ : Due to Theorem 2.2 and condition (a), for sufficiently large $t>0$ we have $\mu\left(\varphi^{t}(S), 2\right)<\mu(S, 2)$. But this is in contradiction to the fact that the membrane $S$ was taken to be of minimal two-dimensional Hausdorff measure.

Now, consider the case $b_{1} \geq 1$. Suppose that (4.1) possesses more than $b_{1}$ closed non-trivial orbits in $M$. Then there are at least two orbits $\gamma_{1}$ and $\gamma_{2}$ among them which are homologous to each other. Let $S$ be a surface of minimal two-dimensional Hausdorff measure with boundary $\gamma_{1} \cup \gamma_{2}$. Then again, for arbitrary $t>0$ the set $\varphi^{t}(S)$ is a surface in $M$ with $\gamma_{1} \cup \gamma_{2}$ as boundary. For sufficiently large $t>0$ the same argument as above together with Theorem 2.2 leads to a contradiction 
Let us now add a version of Theorem 4.1 for the case $n=2$ that in principle agrees with the classical negative Bendixson-Dulac criterion. The difference to Theorem 4.1 is actually that here a modification of the vector field is. allowed in the way that products $\alpha f$ are considered with a $C^{1}$-smooth function $\alpha: M \rightarrow \mathbb{R}$. In the proof it is confirmed that the introduction of the function $\alpha$ can be interpreted, similar to the methods of the previous sections, as a transition to an equivalent Riemannian metric on $M$. In a second corollary a result of [17] is generalized to Riemannian manifolds.

Corollary 4.1.1. Let the manifold $M$ with Betti number $b_{1}$ be two-dimensional and compact. Assume that a function $\alpha: M \rightarrow \mathbb{R}$ of class $C^{1}$ exists such that the divergence $\operatorname{div}(\alpha f)$ does not vanish on $M$. Then the system (4.1) possesses on $M$ at most $b_{1}$ non-trivial periodic orbits.

Proof. We pass on $M$ to the new Riemannian metric $\tilde{g}_{\mid u}:=\alpha(u) g_{\mid u}$ for $u \in M$. Consider the two eigenvalues $\tilde{\lambda}_{1}(u) \geq \tilde{\lambda}_{2}(u)$ of the symmetric part of the covariant derivative of $f$ at $u \in M$ in the new metric $\tilde{g}$. Then we have $\widetilde{\operatorname{div}} f(u)=\tilde{\lambda}_{1}(u)+\tilde{\lambda}_{2}(u)$ for the divergence of $f$ with respect to $\tilde{g}$. On the other hard, a straightforward calculation using formula (3.6) gives

$$
\widetilde{\operatorname{div}} f=\operatorname{div} f+\frac{\dot{\alpha}}{\alpha}=\frac{1}{\alpha} \operatorname{div}(\alpha f) .
$$

When combining relation (3.14) with the last result it becomes clear that one of the conditions (a) or (b) of Theorem 4.1 are satisfied if the inequality $\operatorname{div} f>0$ or $\operatorname{div} f<0$ holds on $M$, respectively

Corollary 4.1.2. Let the manifold $M$ with Betti number $b_{1}$ be compact and suppose that there exists a continuous function $\theta: M \rightarrow \mathbb{R}$ such that for the symmetric part $S$ of $\nabla f$ one of the following conditions is satisfied: $\left.T_{\mathbf{u}} M\right)$.

(a) $\langle S(u) v, v\rangle_{T_{u} M} \geq-\theta(u)\langle v, v\rangle_{T_{u} M}$ and $\operatorname{div} f(u)+(n-2) \theta(u)<0^{\circ} \quad(u \in M, v \in$

(b) $\langle S(u) v, v\rangle_{T_{u} M} \leq \theta(u)\langle v, v\rangle_{T_{u} M}$ and $\operatorname{div} f(u)-(n-2) \theta(u)>0 \quad(u \in M, v \in$ $\left.T_{u} M\right)$.

Then the system: (4.1) possesses on $M$ at most $b_{1}$ non-trivial periodic orbits.

Proof. Again we only prove the case of condition (a). For the second case the same method can be applied. Analogously to Corollary 3.1 .2 we obtain $\lambda_{1}(u)+\lambda_{2}(u) \leq$ $(n-2) \theta+\operatorname{div} f<0$ and this coincides with condition (a) of Theorem 4.1

We want to add a further corollary for the special case that the manifold has the Betti'number $b_{1}=0$. It demonstrates what kind of convergence behaviour a system (4.1) satisfying the assumptions of Theorem 4.1 in this situation necessarily exhibits.

Corollary 4.1.3. Let the manifold $M$ be compact and $\dot{b}_{1}=0$. Let the set of equilibria of system (4.1) consists of isolated points only. If one of the conditions (a) or (b) of Theorem 4.1 holds, then every orbit of system. (4.1) converges both for $t \rightarrow \infty$ and for $t \rightarrow-\infty$ to an equilibrium point.

In order to prove the last result we need the closing lemma by C. C. Pugh [16]. We denote by $X^{1}(M)$ the topological space of vector fields of class $C^{1}$ on $M$ generated by the $C^{1}$-metric. 
Lemma 4.1. Let $p \in M$ be a non-wandering point of system (4.1) such that $f(p) \neq$ 0 . Then in every neighbourhood of $f$ in $X^{1}(M)$ there can be found a vector field $\tilde{f}$ such that the system $\dot{u}=\tilde{f}(u)$ has a non-trivial periodic orbit through $p$.

Proof of Corollary 4.1.3. Again we restrict ourself to the case of condition (a). Consider an arbitrary integral curve $\varphi(\cdot, q)$ of system (4.1) for $t \rightarrow \infty$. The manifold is compact and therefore the $\omega$-limit set $\omega(q)$ is not empty. If we assume that there exists an element $p \in \omega(q)$ such that $p$ is not an equilibrium point for (4.1), then by Lemma 4.1 in every small neighbourhood of $f$ in $X^{1}(M)$ we can find a vector field $\tilde{f}$ such that the corresponding differential equation possesses a non-trivial periodic orbit through $p$.

The compactness of $M$ implies that $\tilde{f}$ can be chosen such that for the first two eigenvalues $\tilde{\lambda}_{1}(u) \geq \tilde{\lambda}_{2}(u)$ of the symmetric part of the covariant derivative of $\tilde{f}$ the property $\tilde{\lambda}_{1}(u)+\tilde{\lambda}_{2}(u)<0$ is maintained on $M$. But this contradicts the statement of Theorem 4.1. Thus, we can conclude that $p$ has to be an equilibrium point of the original system (4.1). Remember that the set of all equilibria of (4.1) was assumed to consist of isolated points only. This finally gives $\omega(q)=\{p\}$, or with other words, the considered integral curve converges for $t \rightarrow \infty$ to $p$.

It is clear, that the convergence for $t \rightarrow-\infty$ follows in analogous manner when investigating the $\alpha$-limit set instead

\section{References}

[1] Abraham, R., Marsden, J.E. and T. Ratiu: Manifolds, Tensor-Analysis, and Applications. New York: Springer-Verlag 1988.

[2] Boichenko, A. V. and G. A. Leonov: Lyapunov's direct method in the estimation of the Hausdorff dimension of attractors. Acta Appl. Math. 26 (1992), 1 - 60.

[3] Butler, G., Schmid, R. and P. Waltman: Limiting the complexity of limit sets in selfregulating systems. J. Math. Anal. Appl. 147 (1990), 63 - 68.

[4] Douady, A. and J. Oesterlé: Dimension de Hausdorff des attracteurs. C.R. Acad. Sci. Paris Ser. A 290 (1980), 1135 - 1138.

[5] Eden, A., Foias, C. and R. Temam: Local and global Lyapunov exponents. J. Dyn. Diff. Equ. 3 (1991), 133 - 177.

[6] Federer, H.: Geometric Measure Theory. New York: Springer-Verlag 1969.

[7] Ledrappier, F.: Some relations between dimension and Lyapunov exponents. Commun. Math. Phys. 81 (1981), $229-238$.

[8] Leonov, G. A., Burkin, I. M. and A. I. Shepeljawyi: Frequency Methods in Oscillating Theory (Mathematics and Its Application: Vol 357). Dortrecht - Boston - London: Kluwer Acad. Publ. 1996.

[9] Leonov, G. A., Reitmann, V. and V. B. Smirnova: Non-Local Methods for PendulumLike Feedback Systems (Teubner-Texte zur Mathematik: Vol. 132). Stuttgart - Leipzig: B. G. Teubner Verlagsges. 1992.

[10] Leonov, W. G.: Estimations of Hausdorff dimension of invariant sets in cylindric phase space. J. Diff. Equ. 30 (1994), 1274 - 1276. 
(11) Marschall, A. W. and I. Olkin: Inequalities: Theory of Majorization and Its Applications. New York: Academic Press 1979.

[12] Li, Yi and J. S. Muldowney: On Bendixson's criterion. J. Diff. Equ. 106 (1993), 27 - 39.

[13] Morrey, C: The problem of Plateau on a Riemannian manifold. Ann. Math. 49 (1948), $807-851$.

[14] Muldowney, J. S.: Compound matrices and ordinary differential equations. Rocky Mountain J. Math. 20 (1990), $857-871$.

[15] Noack, A: Hausdorff dimension estimates for time-discrete feedback control systems. Preprint. DFG-Schwerpunkt-Programm: "Dynamik: Analysis, effiziente Simulation und Ergodentheorie", Preprint 13/95 (1995).

[16] Pugh, C. C: An improved closing lemma and a general density theorem. Amer. J. Math. 89 (1967), 1010 - 1021.

[17] Smith, R. A: Some applications of Hausdorf dimension inequalities for ordinary differential equations. Proc. Roy. Soc. Edinburgh 104A (1986), 235 - 259.

[18] Temam, R: Infinite-Dimensional Dynamical Systems in Mechanics and Physics. New York: Springer-Verlag 1988.

Added in proof:

[19] Lloyd, N. G.: A note on the number of limit cycles in certain two-dimensional systems. J. London Math. Soc. (2) 20 (1979), 277 - 287.

Received 09.08.1995 